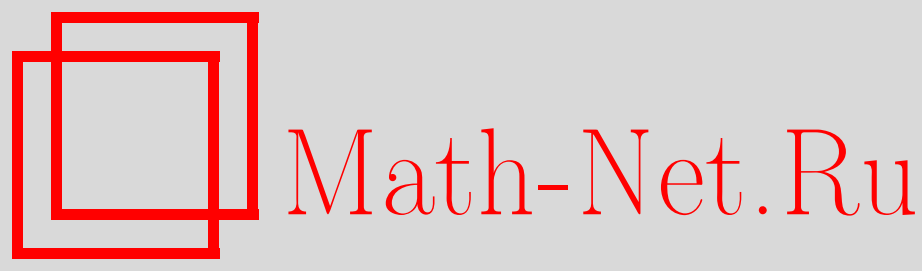

А. Кунду, Интегрируемая двукратная иерархия возмущенных уравнений и ее применение к динамике оптических солитонов, ТМФ, 2011, том 167, номер 3, 465-478

DOI: https://doi.org/10.4213/tmf6654

Использование Общероссийского математического портала Math-Net.Ru подразумевает, что вы прочитали и согласны с пользовательским соглашением http://www . mathnet.ru/rus/agreement

Параметры загрузки:

IP: 54.210 .77 .194

26 апреля 2023 г., $17: 38: 24$

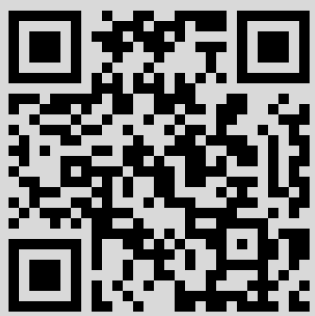




\title{
ИНТЕГРИРУЕМАЯ ДВУКРАТНАЯ ИЕРАРХИЯ ВОЗМУЩЕННЫХ УРАВНЕНИЙ И ЕЕ ПРИМЕНЕНИЕ К ДИНАМИКЕ ОПТИЧЕСКИХ СОЛИТОНОВ
}

\begin{abstract}
С использованием схемы делинеаризации из линейных уравнений построены соответствующие хорошо известные интегрируемые уравнения, а также пара Лакса. С помощью деформирования временно́го оператора Лакса с применением отрицательных степеней спектрального параметра найден класс возмущений, которые, в отличие от обычных возмущений, нарушающих интегрируемость системы, дают двойные интегрируемые иерархии, в частности иерархии для уравнения КдФ, модифицированного уравнения КдФ, уравнения синус-Гордон, нелинейного уравнения Шредингера и нелинейного уравнения Шредингера с производными. Выявлены скрытые возможности применения возмущенной иерархии нелинейного уравнения Шредингера к усилению и управлению оптическими солитонами, распространяющимися через волокно в активированной нелинейной резонансной среде.
\end{abstract}

Ключевые слова: пара Лакса, делинеаризация линейного уравнения, неголономная деформация, оптический солитон, система "нелинейное уравнение Шредингера-уравнение теории самоиндуцированной прозрачности", интегрируемая иерархия.

\section{1. ВВЕДЕНИЕ}

Нелинейные интегрируемые системы прошли впечатляющий путь от своего теоретического открытия до имеющих огромную важность практических применений [1]. Однако, как оказалось, в этой хорошо исследованной области остается пока гораздо больше неоткрытых сегментов, представляющихся очень перспективными с точки зрения приложений. Одной из таких областей является неголономная деформация интегрируемых систем [2], которая приводит к интегрируемым возмущениям известных интегрируемых систем с двойной иерархией и более богатой динамикой солитонов [3], а также к открытию новых уравнений таких, как интегрируемые возму-

${ }^{*}$ Theory Group \& CAMCS, Saha Institute of Nuclear Physics, Calcutta, India. E-mail: anjan.kundu@saha.ac.in 
щения модифицированного уравнения КдФ (мКдФ), уравнения синус-Гордон (СГ), нелинейного уравнения Шредингера (НУШ) и НУШ с производными (ДНУШ), и к получению некоторых недавно предложенных уравнений, например уравнения КдФ6 [4], уравнения Леннелса-Фокаса (ЛФ) [5] и т. д. Кроме того, использование неголономной деформации позволяет объяснить интегрируемость данных уравнений и получить точные солитоные решения [3], [6]-[8].

Интегрируемые уравнения всегда обладают определенным типом нелинейности (мы будем ее называть интегрируемой нелинейностью), малейшее изменение которой нарушает интегрируемость. Интегрируемые уравнения в большинстве случаев связаны с такими богатыми структурами, как пара Лакса, бесконечное множество сохраняющихся величин, а также интегрируемая иерархия уравнений высших порядков и точные устойчивые солитонные решения, устойчивость которых, как считается, обусловлена тонким балансом между линейным дисперсионным и интегрируемым нелинейным членами.

Возникает интригующий вопрос: можно ли, зная только линейный дисперсионный член, единственным образом построить интегрируемую нелинейность для уравнений, относящихся к классу уравнений Абловица-Каупа-Ньюэлла-Сегуры (АКНС) и Каупа-Ньюэлла (KН). В пользу такой идеи высказывались, предлагая разнообразные изощренные методы, такие стойкие ее приверженцы, как Сато, Абловиц, Кауп, Ньюэлл, Сегур, Захаров, Шабат, Калоджеро, Дегасперис и другие.

Мы предлагаем, вероятно, простейшую схему делинеаризации линейных уравнений, основанную на анализе физического понятия скейлинговой размерности [3]. Используя данный метод, мы смогли наиболее просто единственным образом построить пару Лакса для генерации таких хорошо известных уравнений, как уравнения КдФ, мКдФ, НУШ, СГ, ДНУШ и т. д. При этом дальнейшая деформация временно́го оператора Лакса посредством использования отрицательных степеней спектрального параметра может порождать для этих хорошо известных интегрируемых уравнений новые интегрируемые возмущения с неголономными связями. Этот новый класс деформированных интегрируемых уравнений, обладающих более интересными свойствами, например двукратной иерархией и точными решениями типа ускоряющихся солитонов с изменением формы [3], [6]-[8], важен не только с теоретической точки зрения, но и с точки зрения приложений.

Одно из таких применений деформированного НУШ к волоконно-оптическому обмену информацией, описываемому солитонными решениями, составляет основной новый результат настоящей работы. Основное деформированное НУШ можно соотнести со связанной системой НУШ-уравнения теории самоиндуцированной прозрачности (СИП), применяемой при описании волоконно-оптического обмена информацией через среду, активированную эрбием. Но при этом осознание того, что столь важное уравнение действительно связано с неголономной деформационной иерархией НУШ, побудило нас исследовать ценные применения, которые рассмотрены в настоящей работе. Кроме того, мы представляем систематический, хотя и краткий обзор нашей схемы делинеаризации и других результатов, связанных с двойной неголономной деформационной иерархией интегрируемого возмущения, приводящей к ускоряющемуся точному солитонному решению. 


\section{2. НЕЛИНЕЙНЫЕ ИНТЕГРИРУЕМЫЕ СИСТЕМЫ}

Начнем с примеров наиболее популярных интегрируемых систем в размерности $(1+1)$ :

$$
\begin{array}{lrlrl}
\text { НУШ: } & i q_{t}-q_{x x}+2|q|^{2} q & =0, & i q_{t}^{*}+q_{x x}^{*}-2|q|^{2} q^{*}=0, \\
\text { ДНУШ: } & i q_{t}-q_{x x}+2\left(|q|^{2} q\right)_{x} & =0, & i q_{t}^{*}+q_{x x}^{*}-2\left(|q|^{2} q^{*}\right)_{x}=0, \\
\text { КдФ: } & u_{t}-u_{x x x}+6 u u_{x} & =0, & \\
\text { мКдФ: } & u_{t}-u_{x x x}+6 u^{2} u_{x} & =0, \\
\text { СГ: } & \theta_{x t}-\sin \theta & =0 . &
\end{array}
$$

Заметим, что во всех приведенных эволюционных уравнениях имеется линейный член, который является дисперсионным, и специальный нелинейный член, добавление которого делает систему интегрируемой. Мы покажем, что, зная только линейные члены, можно единственным образом построить соответствующие нелинейные члены, используя при этом лишь несколько дополнительных входных данных, а также можно генерировать пертурбативный член с дополнительными неголономными связями, сохраняющими интегрируемость системы.

Напомним некоторые общие, но замечательные свойства интегрируемых систем. Большинство нелинейных интегрируемых уравнений можно ассоциировать с линейной системой, задаваемой уравнениями Лакса

$$
\Phi_{x}=U(\lambda) \Phi, \quad \Phi_{t}=V_{n}(\lambda) \Phi
$$

с парой Лакса $\left(U(\lambda), V_{n}(\lambda)\right)$ и условием нулевой кривизны

$$
U_{t}(\lambda)-V_{n, x}(\lambda)+\left[U(\lambda), V_{n}(\lambda)\right]=0,
$$

которое приводит к интегрируемой иерархиии нелинейных уравнений при $n=1,2, \ldots$; некоторые типичные примеры таких уравнений при фиксированных значениях $n$ приведены выше. Временной оператор Лакса $V_{n}(\lambda)$ обычно представляет собой многочлен порядка $n$ по положительным степеням спектрального параметра $\lambda$. Такая иерархия уравнений отвечает бесконечному множеству сохраняющихся величин $C_{n}$ в инволюции: $\left[C_{n}, C_{m}\right]=0$.

Другим важным универсальным свойством интегрируемых систем является существование точного, локализованного, сохраняющего форму и скорость солитонного решения, устойчивость которого, как предполагается, обусловлена балансом между линейным дисперсионным и нелинейным членами. Поэтому линейный член должен содержать скрытую информацию об интегрируемом нелинейном члене, и это побудило нас разработать прямую схему делинеаризации.

\section{3. СХЕМА ДЕЛИНЕАРИЗАЦИИ}

Наша схема основана на простом принципе скейлинговой размерности и отождествлении тех основных элементов, из которых построены пары Лакса. Заметим, что уравнения Лакса (2) определяют размерность (обозначаемую через [·]) пары 
Лакса $\left(U, V_{n}\right)$ как $[U]=\left[L^{-1}\right] \equiv 1,\left[V_{n}\right]=\left[T^{-1}\right]$. Возьмем в качестве исходных линейные уравнения

$$
\begin{gathered}
i q_{t}-q_{x x}=0, \quad i q_{t}^{*}+q_{x x}^{*}=0, \\
u_{t}-u_{x x x}=0, \\
\theta_{x t}=0
\end{gathered}
$$

которые представляют собой линейные дисперсионные части интегрируемых уравнений (1). Покажем, что эти линейные уравнения можно делинеаризовать, сделав несколько простых шагов, позволяющих единственным образом воспроизвести полный набор интегрируемых уравнений.

На первом шаге заметим, что линейные уравнения (4), (5) и (6) фиксируют скейлинговые размерности $\left[V_{n}(\lambda)\right]=\left[T^{-1}\right]$ как $\left[L^{-2}\right]=2,\left[L^{-3}\right]=3,\left[L^{-1}\right]=1$ соответственно. Предположим далее, что пара Лакса также должна иметь вид

$$
U(\lambda)=U_{\mathrm{L}}(\lambda)+U_{\mathrm{NL}}(\lambda), \quad V_{n}(\lambda)=V_{\mathrm{L}}(\lambda)+V_{\mathrm{NL}}(\lambda),
$$

где наивная линейная пара Лакса $\left(U_{\mathrm{L}}, V_{\mathrm{L}}\right)$ удовлетворяет линейной части условия нулевой кривизны (3) (без нелинейного члена в квадратных скобках): $\left(U_{\mathrm{L}}\right)_{t}-\left(V_{\mathrm{L}}\right)_{x}=$ 0 , что дает линейные уравнения (4)-(6).

Опуская детали для каждого из приведенных выше уравнений, покажем, как работает наша схема лишь на примере линейного уравнения Шредингера (4), которое, очевидно, дает

$$
U_{\mathrm{L}}(\lambda)=i\left(\lambda \sigma_{3}+U^{(0)}\right), \quad V_{\mathrm{L}}=\sigma^{3} U_{x}^{(0)}, \quad U^{(0)}=\left(\begin{array}{cc}
0 & q \\
q^{*} & 0
\end{array}\right)
$$

где $\lambda$ - постоянный параметр, а матрица Паули $\sigma^{3}$ обеспечивает выполение условия $\operatorname{tr} U=0, \operatorname{tr} V=0$. Несложно проверить, что линейное условие нулевой кривизны $i U_{t}^{(0)}-\sigma^{3} U_{x x}^{(0)}=0$ воспроизводит уравнение (4).

Следующий шаг состоит в том, чтобы задать основные элементы, из которых строятся пары Лакса $(7)$, в виде $\lambda, U^{(0)}$ и $x$-производных типа $U_{x}^{(0)}$. Используя их с подходящим скейлинговым весом, мы намереваемся построить нелинейную дополнительную часть $U_{\mathrm{NL}}(\lambda)$, чтобы получить пару настоящих операторов Лакса $\left(U(\lambda), V_{n}(\lambda)\right)$ для интегрируемой системы.

Поскольку $[U(\lambda)]=\left[U_{\mathrm{L}}(\lambda)\right]=1$, имеем $[\lambda]=\left[U^{(0)}\right]=[q]=\left[q^{*}\right]=1,\left[U_{x}^{(0)}\right]=2$ и т. д., откуда мы заключаем, что пространственный оператор Лакса с $[U]=1$ должен единственным образом задаваться как

$$
U(\lambda) \equiv U_{\mathrm{L}}=i\left(\lambda \sigma^{3}+U^{(0)}\right),
$$

ибо нет других операторов размерности 1, построенных из основных элементов. Для временно́го оператора Лакса $V_{2}(\lambda)$ с $\left[V_{2}\right]=2$, кроме тех же самых основных элементов $\left(\lambda, U^{(0)}\right.$, где $\left.\left[U^{(0)}\right]=1\right)$, мы использовали их комбинации, образованные произведениями, степенями и производными, что дает

$$
V_{2}(\lambda)=V_{\mathrm{L}}+\underbrace{\left(2 i \lambda^{2}-\left(U^{(0)}\right)^{2}\right) \sigma^{3}+2 i \lambda U^{(0)}}_{V_{\mathrm{NL}}}
$$


Замечательно, что коэффициенты единственным образом фиксированы условием нулевой кривизны (3), и невозможно построить никакого другого оператора размерности 2.

Таким образом, с помощью простого размерностного анализа мы построили точную пару Лакса для НУШ, условие нулевой кривизны которой дает нелинейное уравнение: к исходному линейному уравнению добавляется интегрируемый нелинейный член $2\left(U^{(0)}\right)^{3} \rightarrow 2|q|^{2} q$. Это завершает нашу схему делинеаризации, которая представляет прототип всего семейства уравнений АКНС-Захарова-Шабата с тем же $U(\lambda)$. Можно продолжить построение высших операторов $V_{n},\left[V_{n}\right]=n$, из тех же основных элементов, чтобы вывести иерархию НУШ, причем случай $\left[V_{3}\right]=3$ приводит к уравнениям КдФ и мКдФ.

Отметим, что нашу схему делинеаризации также можно распространить на семейство уравнений $\mathrm{KH}$, задавая новую скейлинговую размерность $[q]=[\lambda]=1 / 2$. Теперь нам нужно построить соответствующую пару Лакса с $[U(\lambda)]=1,\left[V_{2}(\lambda)\right]=2$ из тех же основных элементов $\left(\lambda, U^{(0)}\right.$ и его $x$-производные), но с новой скейлинговой размерностью поля $q$ и параметра $\lambda$. Это должно естественным образом привести к

$$
\begin{aligned}
U(\lambda) & =i\left(\lambda U^{(0)}+\lambda^{2} \sigma^{3}\right), \\
V_{2}(\lambda) & =\lambda\left(\sigma^{3} U_{x}^{(0)}+i\left(U^{(0)}\right)^{3}\right)-i \sigma^{3}\left(U^{(0)}\right)^{2} \lambda^{2}+2 i U^{(0)} \lambda^{3}+2 i \sigma^{3} \lambda^{4},
\end{aligned}
$$

откуда получаем не что иное, как пару Лакса для ДНУШ и, с помощью условия (3), ДНУШ $i q_{t}-q_{x x}+2 i\left(|q|^{2} q\right)_{x}=0$ из того же линейного уравнения $i q_{t}-q_{x x}=0$. Заметим, что наша схема делинеаризации определенно является простейшим алгоритмическим методом построения пары Лакса $\left(U, V_{n}\right)$ для систем уравнений АКНС и $\mathrm{KH}$, основаным на базовой физической концепции скейлинговой размерности.

\section{4. ИНТЕГРИРУЕМЫЕ ВОЗМУЩЕНИЯ С ИСПОЛЬЗОВАНИЕМ НЕГОЛОНОМНОЙ ДЕФОРМАЦИИ}

Заметим, что в приведенной выше конструкции для временно́го оператора Лакса мы рассмотрели только члены с положительными степенями $\lambda$. Однако мы могли бы также рассмотреть члены с отрицательными степенями, добавляя в оператор Лакса с $\left[V_{2}(\lambda)\right]=2$, например, члены с более высокой скейлинговой размерностью вида $\lambda^{-1} G_{(1)},\left[G_{(1)}\right]=3$, где $G_{(1)}$ содержит возмущающие поля с более высокой скейлинговой размерностью (т. е. мы не ограничиваемся основными элементами, вводя дополнительные поля). В этом и заключается идея, лежащая в основе интересуюшего нас интегрируемого возмущения. В результате мы получим известные интегрируемые уравнения, возмущенные функцией $g(x, t)$, которая подчиняется неголономной связи, т. е. связи, которая включает в себя функцию $g$ и ее производные (импульс), что оправдывает название "неголономная связь". Напомним, что возмущение, как правило, разрушает интегрируемость, однако это не так для интегрируемых возмущений. Покажем, что такие возмущения не только сохраняют интегрируемость пары Лакса, но также обладают богатой структурой с двойной интегрируемой иерархией, ускоряющимися точными солитонными решениями и важными возможными применениями. Хотя эти свойства являются общими для всех возмущенных интегрируемых систем, обсуждаемых ниже, мы подробно рассмотрим только НУШ, 


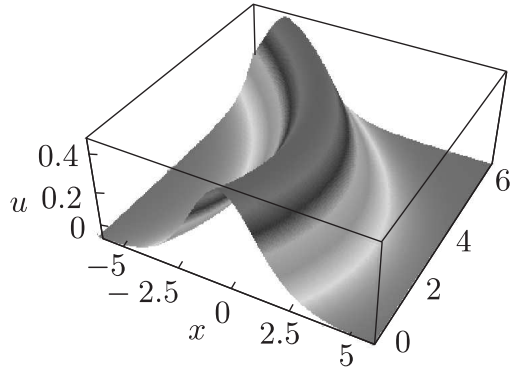

a

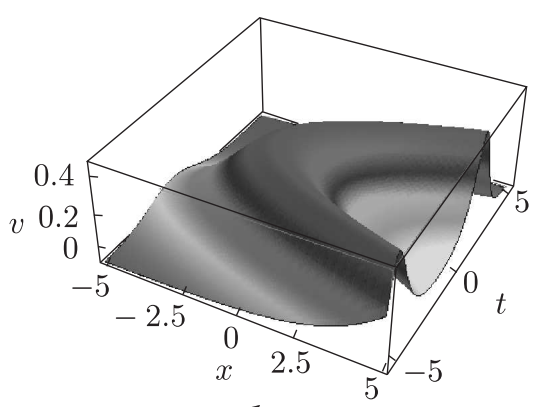

б

Рис. 1. Точный ускоряющийся солитон $u(x-V(t))$ для интегрируемого возмущения уравнения КдФ (а) и точный ускоряющийся солитон $v(x-V(t))$ для интегрируемого возмущения уравнения мКдФ (б). Изгиб солитона в плоскости $(x, t)$ показывает его ускорение со скоростью солитона $V(t)$.

поскольку аналогичные свойства других уравнений можно получить, следуя тем же путем.

Заметим, что идея интегрируемого возмущения была предложена в работе [9], однако она слишком сложна для любого практического применения и, в отличие от нашего случая, не допускает никакой интегрируемой иерархии. С другой стороны, кроме хорошо известных уравнений Камассы-Холма и Дегаспериса-Прочези, недавно были открыты и другие интегрируемые уравнения, а именно уравнения КдФ6 [4], ДНУШ-ЛФ [5], мКдФ-СГ [10] и высшее мКдФ [11]. Мы осознали, что эти необычные уравнения являются не чем иным, как частными случаями общих интегрируемых возмущений для систем уравнений АКНС-Захарова-Шабата и КН [6]-[8].

Приведем краткое описание возмущенных интегрируемых систем таких, как возмущенные НУШ, уравнения КдФ, мКдФ, СГ и ДНУШ.

4.1. Интегрируемое возмущение НУШ. Возмущенное НУШ можно записать в виде

$$
i q_{t}-q_{x x}-2|q|^{2} q=g
$$

где $g(x, t)$ - возмущающая функция, подчиняющаяся нелинейной неголономной связи

$$
g_{x x} q-g_{x} q_{x}-2 q^{2}\left(q g^{*}-q^{*} g\right)=0 .
$$

Ниже будет подробно рассмотрено физическое применение этой системы, которой мы уделяем основное внимание в настоящей работе. Солитоное решение возмущенного НУШ, выраженное через $|q(x, t)|$, аналогично решению уравнения мКдФ (см. рис. 1б, где показано появление зависящей от времени скорости солитона $V(t))$.

Двойная интегрируемая иерархия. Хотя это важное свойство описывается здесь только применительно к возмущенному НУШ, оно в равной степени справедливо для всех других возмущенных уравнений, которые мы будем рассматривать 
ниже. Первую иерархию представляют стандартные НУШ высшего порядка с возмущением:

$$
U_{t}-V_{n, x}+\left[U, V_{n}\right]=\left[\sigma^{3}, G^{(1)}\right] .
$$

Здесь $n=1,2,3, \ldots$ дают НУШ, уравнение Хироты и НУШ высшего порядка в иерархии с той же связью (9). С другой стороны, вторую иерархию можно задать с помощью того же НУШ (8), но с иерархией условий связи для деформирующей функции $g(x, t)$. Такую иерархию можно получить, деформируя временной оператор Лакса невозмущенного НУШ, полученного выше, с дополнительным членом типа $V_{\text {def }}=\sum_{n=1}^{N} \lambda^{-n} G_{(n)}$, где деформирующая матрица $G_{(n)}$ представляет собой матрицу высшей скейлинговой размерности $\left[G_{(n)}\right]=(2+n)$, задаваемую рекуррентно с помощью неголономных связей:

$$
\begin{aligned}
G_{x}^{(1)} & =i\left[U^{(0)}, G^{(1)}\right]+i\left[\sigma_{3}, G^{(2)}\right], \quad \ldots, \\
G_{x}^{(N-1)} & =i\left[U^{(0)}, G^{(N-1)}\right]+i\left[\sigma_{3}, G^{(N)}\right], \\
G_{x}^{(N)} & =i\left[U^{(0)}, G^{(N)}\right] .
\end{aligned}
$$

В следующем разделе мы продемонстрируем интересное применение такой двойной возмущенной иерархии НУШ.

4.2. Интегрируемое возмущение уравнения КдФ. Уравнение КдФ возмущается функцией $g(x, t)=w_{x}$,

$$
u_{t}-u_{x x x}-6 u u_{x}=g
$$

с неголономной связью

$$
w_{x x x}+4 u w_{x}+2 u_{x} w=0
$$

что эквивалентно недавно открытому уравнению КдФ6 [4]. Такую систему можно расширить до двойной иерархии; кроме того, она обладает точным решением в виде ускоряющегося солитона [8] (см. рис. 1а).

4.3. Интегрируемое возмущение уравнения мКдФ. Система задается с помощью возмущенного уравнения мКд $\Phi$

$$
v_{t}-v_{x x x}-6 v^{2} v_{x}=w_{x}
$$

со связью

$$
w_{x x}-2 v\left(c^{2}(t)-w_{x}^{2}\right)^{1 / 2}=0 .
$$

Ускоряющийся солитон показан на рис. 1б, система обладает двойной интегрируемой иерархией и интересной симметрией [6].

4.4. Интегрируемое возмущение уравнения СГ. Интегрируемое возмущение для такой системы проявляется интересным образом, когда наряду с синусом также дополнительно появляется член, содержащий косинус:

$$
\theta_{x t}=e(t)(\alpha \sin 2 \theta+\beta \cos 2 \theta),
$$

где $e(t)$ - произвольная зависящая от времени функция, а массовому параметру теперь соответствуют возмущающие функции $\alpha, \beta$, удовлетворяющие условиям связи

$$
\alpha_{x}=\sin 2 \theta, \quad \beta_{x}=\cos 2 \theta .
$$

Ускоряющийся кинковый солитон показан на рис. 2 a. 


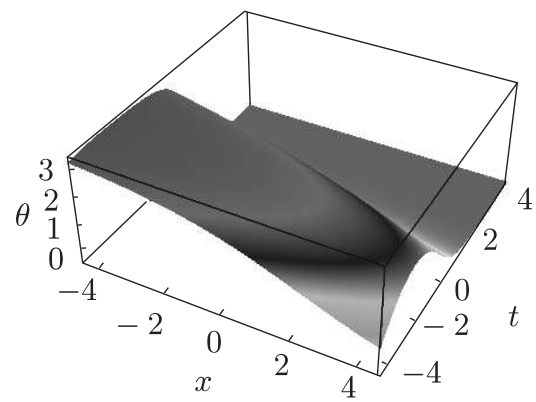

a

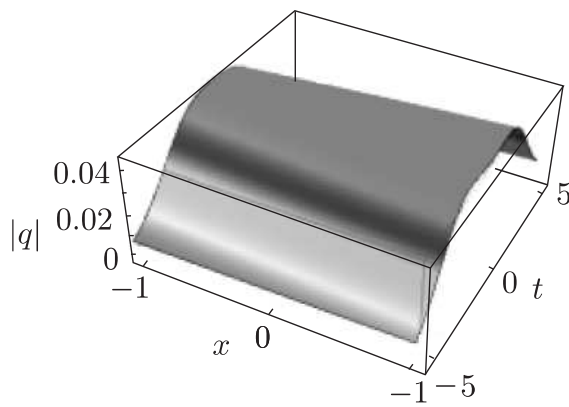

6

Рис. 2. Точный ускоряющийся солитон $\theta(x-V(t))$ с кинковым профилем в интегрируемом возмущенном уравнении СГ (а) и точный ускоряющийся солитон $|q(x-V(t))|$ с солитонным профилем в интегрируемом возмущенном ДНУШ (б).

4.5. Интегрируемое возмущение ДНУШ. Наконец, получим возмущенное ДНУШ с богатой интегрируемой структурой:

$$
\frac{i}{2} q_{t}-q_{x x}+2 i \epsilon\left(|q|^{2} q\right)_{x}+(-2 w+\epsilon g q)=0, \quad \epsilon= \pm 1 .
$$

Возмущающие функции $w(x, t), g(x, t)$ подчиняются условиям связи

$$
g_{x}(x, t)=i \epsilon\left(q w^{*}-q^{*} w\right), \quad w_{x}(x, t)=2 i a(t) q .
$$

Такое интегрируемое ДНУШ допускает точное решение типа ускоряющегося $n$-солитона, а также порождает двойную иерархию, как и в описанном выше случае [7]. Возможное применение такой системы в нелинейной оптике для описания распространения солитонного импульса через активированную среду сейчас усиленно нами исследуется, и ему будет посвящена отдельная работа.

Мы замечаем, что предложенное недавно уравнение ЛФ представляет собой низший член иерархии ДНУШ, задаваемый в виде (где мы положили $q=u_{x}$ )

$$
\left(\frac{i}{2} u_{x t}+a u\right)-\alpha u_{x x}+c u_{x}+\epsilon a|u|^{2} u_{x}=0 .
$$

Если коэффициенты $a(t), c(t)$ зависят от времени, то мы получаем неавтономное обобщение [7] уравнения ЛФ, допускающее решения типа ускоряющийся солитон, как на рис. 2 б.

\section{5. ПРИМЕНЕНИЕ К НЕЛИНЕЙНОЙ ОПТИКЕ}

Волоконно-оптические коммуникации стали интенсивно исследоваться теоретически и экспериментально в связи с их огромной практической и коммерческой значимостью [12]. Диссипация и дисперсия в среде являются основными помехами при прохождении сигнала через оптические волокна [13]. Чтобы противодействовать 
этому, первоначально был предложен способ коммуникации на основе солитонного решения НУШ [14], когда дисперсию групповой скорости в световом импульсном волноводе можно уравновесить фазовой самомодуляцией в нелинейно-волоконной среде [13]. Другое важное предложение основано на явлении СИП, создаваемой когерентным откликом среды на ультракороткий оптический импульс [15], [16]. В работе [17] было предложено объединить преимущества НУШ и СИП, когда распространение устойчивого сигнала может достигаться путем передачи оптического импульса через активированную эрбием $\left(\mathrm{Er}^{3+}\right)$ нелинейную резонансную среду, описываемую солитонным решением связанной системы НУШ-СИП [18]-[20] или системы более высокого порядка - уравнением Хироты-СИП [21].

Наша цель - предложить унифицированное описание этих имеющих интерес с точки зрения важных связанных систем в терминах двойной возмущенной интегрируемой иерархии НУШ, введенной в предыдущем разделе, и выявить особенности, скрытые в системе НУШ-СИП, используя точное аналитическое рассмотрение. Это может дать нам новые возможности усиления и управления солитонным сигналом, а также позволит заменить систему СИП на кратную систему с улучшенными показателями. Следуя обозначениям, принятым в нелинейной оптике, перепишем возмущенное НУШ (8) в виде

$$
i E_{z}=E_{t t}+2|E|^{2} E+\langle p\rangle,
$$

где $E$ - распространяющееся электрическое поле, а $\langle p\rangle \equiv p-$ усредненная поляризация. Неголономная связь (9) может в действительности представлять систему СИП:

$$
p_{t}=i\left(2 N E-w_{0} p\right), \quad N_{t}=-i\left(E p^{*}-E^{*} p\right) .
$$

Индуцированная поляризация, обусловленная двухуровневыми атомами, задается матричным элементом $p=\nu_{1} \nu_{2}^{*}$, а инверсная заселенность $N=\left|\nu_{2}\right|^{2}-\left|\nu_{1}\right|^{2}$, где $-1 \leqslant N \leqslant 1$, описывается нормированными волновыми функциями $\nu_{1}$ и $\nu_{2}$ основного и возбужденного состояний соответственно.

В связанной системе НУШ-СИП (19), (20) оптический импульсный сигнал, входящий в волокно с достаточной интенсивностью и распространяющийся через активированную эрбием резонансную среду, трансформируется в устойчивый солитон:

$$
E=\eta \operatorname{sech} \zeta \cdot e^{i \theta}, \quad \zeta=\eta(t-v z), \quad \theta=\omega z+k t .
$$

Хотя это решение похоже на солитон НУШ, параметры изменяются вследствие взаимодействия со вкладами как от части, отвечающей НУШ, так и от части, отвечающей СИП:

$$
v=v_{\text {нуш }}+v_{\text {Сип }}, \quad \omega=\omega_{\text {нуш }}+\omega_{\text {Сип }}, \quad k \rightarrow \tilde{k}=k-w_{0} .
$$

Заметим, что в отсутствие системы СИП мы должны получить $v_{\text {Сип }}=\omega_{\text {сип }}=$ $w_{0}=0$, что воспроизводит хорошо известный солитон НУШ, в то время как солитон системы СИП можно получить аналогично, если положить $v_{\mathrm{Hуш}}=\omega_{\mathrm{Hуш}}=0$ в $(22)$. С другой стороны, связанная система НУШ-СИП демонстрирует более богатые свойства из-за взаимодействия между двумя своими составляющими, замечательным образом допускающего только существование движущегося солитона. Однако мы обнаружили, что такая богатая картина взаимодействия системы НУШ-СИП 
была неправильно истолкована в известных более ранних работах [18], [19], где, в отличие от соотношений (22), задержка импульса бралась в виде $v=v_{\text {Сип }}$, а вращение фазы - в виде $\omega=\omega_{\text {нуш }}$.

Параметры солитона, связанные с НУШ, можно выразить как

$$
v_{\text {нуш }}=-2 k, \quad \omega_{\text {Нуш }}=k^{2}-\eta^{2} .
$$

Те же параметры, связанные с системой СИП, обычно задаются как [18]-[20]

$$
v_{\text {Сип }}=-\frac{1}{\rho}, \quad \omega_{\text {Сип }}=-\frac{\tilde{k}}{\rho},
$$

где $\rho=\left|\lambda_{1}-w_{0}\right|^{2}=\tilde{k}^{2}+\eta^{2}$. Однако из нашего опыта исследования возмущенной интегрируемой системы [3], [8] мы заключаем, что эти солитонные параметры можно задать в более общем виде, включающем в себя зависящую от $z$ произвольную функцию $c(z) \neq 0$ :

$$
v_{\text {Сип }}=\frac{1}{z \rho} \int^{z} c\left(z^{\prime}\right) d z^{\prime}, \quad \omega_{\text {Сип }}=\tilde{k} v_{\text {Сип }},
$$

где $c(z)$ определяется начальным профилем инверсной заселенности. Ниже мы покажем, что наше обобщение, которое при $c(z)=-1$ сводится к известным выражениям, может играть важную роль в управлении солитонами. Решение для диполя $p$ в связанных уравнениях НУШ-СИП, полученное из (19) с использованием солитонного решения (21) с учетом соотношений (22) и (24), также принимает солитонный вид:

$$
p=\frac{\eta}{\rho} c(z) \operatorname{sech} \zeta \cdot(\tilde{k}-i \eta \operatorname{th} \zeta) e^{i \theta},
$$

где явно появляется $c(z) \neq 0$. Аналогично, инверсную заселенность можно получить в точном виде из системы уравнений (19), (20) с использованием солитонных решений $(21)$ и $(25)$ :

$$
N(z, t)=c(z)\left(1-\frac{\eta^{2}}{\rho} \operatorname{sech}^{2} \zeta\right),
$$

где произвольная ненулевая функция $c(z)=N(z,-\infty)$ появляется как постоянная интегрирования. Это указывает на то, что при ненулевой функции $c(z)>-1$ активирующие атомы должны быть исходно переведены в возбужденное состояние с помощью оптической накачки, что приводит к лазерной активной усиливающей среде, причем интенсивность определяется функцией $c(z)$. Как мы увидим ниже, предложенное общее начальное условие $c(z)>-1$ для системы НУШ-СИП может играть решающую роль при импульсном усилении с использованием начальной энергии накачки, а также является дополнением к новому способу управления формой и динамикой оптического солитона $(21)$. Эти возможности теряются, если традиционно брать исходные атомы в их основных состояниях $\left|\nu_{1}\right|^{2}=1,\left|\nu_{2}\right|^{2}=0$, ограничиваясь случаем $c(z)=-1$.

Произвол в выборе функции $c(z)$ открывает важную возможность решения проблемы уширения импульса за счет регулирования начальной инверсной заселенности активирующих атомов. Например, солитонный импульс, управляемый только 
НУШ, при воздействии пертурбативного члена $-i \Gamma E / 2, \Gamma \ll 1$, должен испытывать затухание за счет множителя $\eta(z)$ и уширение за счет обратного множителя $(2 k \eta(z))^{-1}$, где по теории возмущений можно найти $\eta(z)=\eta e^{-\Gamma z}[13]$. Хотя затухание ведет к потере интенсивности, уширение приводит к более серьезной проблеме потери информации и ограничению частотного диапазона. Поэтому, преобразовав поле $E \rightarrow \eta^{-1}(z) E$, мы сконцентрируемся только на проблеме уширения солитона НУШ, обусловленного увеличением ширины солитона $e^{\Gamma z} / 2 k \eta$ вдоль оси $z$. Замечательным образом мы получили, что при прохождении этого солитонного импульса с возрастающей шириной через активированную резонансную среду, описываемую связанной системой НУШ-СИП (19), (20), можно управлять уширением импульса, если подходящим образом приготовить начальную инверсную заселенность в соответствии с функцией $c(z)$. При этом уширение солитонного импульса, обусловленное $\eta^{-1}(z)$, можно компенсировать, если сделать этот импульс более узким за счет переменной ширины $V(z)=\left(v_{\text {нуш }}+\eta c(z) / \rho\right)^{-1}$, взяв $c(z) \sim \eta^{-1}(z)$. Динамика солитона также меняется с изменением скорости $V(z)$ при ускоренном движении, что, возможно, связано с энергией, которую обеспечивает начальная оптическая накачка. Эти скрытые возможности оставались не изученными в более ранних работах из-за частного выбора $c(z)=-1$.

5.1. НУШ, связанное с кратной системой СИП. Замечательным образом можно достичь значительного увеличения усиления и управления оптическим солитоном в системе НУШ-СИП, если заменить одиночную систему СИП, которая до настоящего времени рассматривалась в литературе, на иерархию систем СИП, используя возмущенную иерархию, представленную в (10). Такая многообещающая возможность была полностью упущена в более ранних исследованиях. Положив $N=2$ в указанной иерархии, получаем деформированное НУШ (19), связанное с кратной системой СИП:

$$
\begin{array}{llrl}
p_{t} & =i\left(2 N E-w_{0} p+e\right), & & N_{t}=-i\left(E p^{*}-E^{*} p\right), \\
e_{t} & =i\left(2 M E-w_{0} e\right), & M_{t} & =-i\left(E e^{*}-E^{*} e\right) .
\end{array}
$$

Здесь мы рассмотрим только две связанные системы СИП с индуцированной поляризацией $е$ и инверсной заселенностью $M$, описывающими вторую систему СИП. Этот процесс можно продолжить вплоть до иерархиии уравнений СИП, представленной в (10), при этом интегрируемость системы не будет нарушена. Интересно, что точное солитонное решение для оптического импульса в этой более общей системе НУШ-СИП можно снова выразить в виде (21), где параметры солитона модифицированы в результате вкладов от всех взаимодействующих частей:

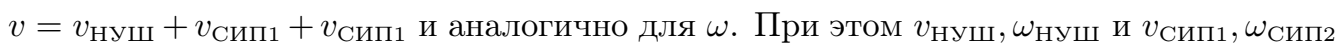
даются теми же выражениями (23) и (24), тогда как вклад от дополнительной системы СИП дается несколько более сложным выражением

$$
v_{\text {Сип2 }}=2 \frac{\tilde{k}}{z \rho^{2}} \int^{z} c_{2}\left(z^{\prime}\right) d z^{\prime}
$$

и аналогично для $\omega_{\text {сип2 }}$. Поэтому ширину и динамику солитона можно регулировать с помощью дополнительного управления, которое обеспечивает вторая система 


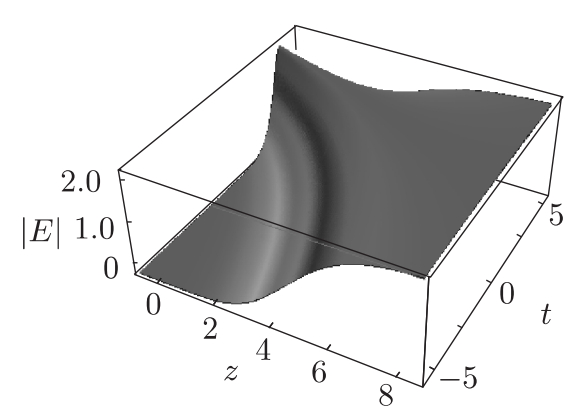

a

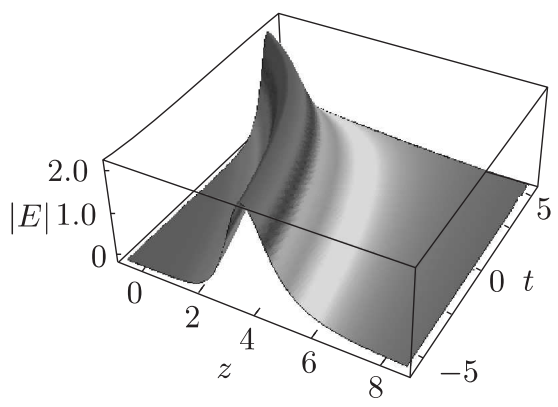

б

Рис. 3. Уширение солитона $|E(z, t)|$ для возмущенного НУШ при его движении вдоль волокна (а) и управляемый солитонный импульс системы НУШ, связанной с двойной системой СИП (б), показывающие реконструкцию ширины солитона и постоянное изменение скорости солитона.

СИП. Эта система аналогичным образом регулируется начальной инверсной заселенностью второго набора активированных атомов, при этом $M(z,-\infty)=c_{2}(z)$. Результаты расчетов приведены на рис. 3. Эти крайне интересные аспекты, относящиеся к приложениям, сейчас изучаются, и мы надеемся рассмотреть их более подробно в отдельной работе.

5.2. Система Хироты-СИП и возмущенная иерархия. Используя временной оператор Лакса $V_{n}$ для иерархии НУШ при $n>2$, можно получить интегрируемые возмущения НУШ высших порядков. В частном случае $n=3$ должна возникать система Хироты-СИП, которая изучалась в работе [21]. Можно естественным образом рассматривать НУШ-СИП и более высоких порядков, если взять $n>3$. Другая возможность возникает, если взять вторую иерархию, допускаемую при интегрируемом возмущении, построив, например, систему Хироты-мультиСИП, зафиксировав $n=3$, но рассматривая при этом высшую возмущенную иерархию (10). Это не только дает универсальное описание класса систем НУШ-СИП, которые уже рассматривались в литературе, но и открывает нам новое богатое семейство подобных связанных систем, использующее двойную интегрируемую иерархию возмущенного НУШ. Продолжение на положительные степени спектрального параметра дает высшие НУШ типа уравнения Хироты, связанные с системой СИП (20), тогда как продолжение на отрицательные степени спектрального параметра дает систему мультиСИП типа (26), связанную с НУШ, уравнением Хироты или высшим НУШ, для которых возможны более эффективные применения в нелинейной оптике. Поэтому с помощью умелого использования интегрируемого возмущения можно предложить полезное применение в такой важной области, как нелинейная оптика, что обещает много новых возможностей для связанной системы НУШ-СИП. Эти возможности включают усиление и управление солитонными импульсами с помощью регулирования начальной инверсной заселенности активирующих атомов в резонансной среде, а также с использованием кратно активированной среды для более впечатляющих достижений. 
Ту же идею можно распространить на все другие представленные выше системы $\mathrm{AKHC} \mathrm{и} \mathrm{KH}$ с потенциальными применениями в других областях.

\section{6. ЗАКЛЮЧИТЕЛЬНЫЕ ЗАМЕЧАНИЯ}

Мы представили схему делинеаризации линейных уравнений для получения интегрируемых систем. Хотя наш подход подобен подходу АКНС, он отличается мотивировкой, простотой и более общим характером. Схема основана на физическом понятии размерностного анализа и отождествлении основных элементов, из которых составлены операторы Лакса, что также позволяет без особых трудностей воспроизвести как семейство уравнений АКНС, так и семейство уравнений КН. Дальнейшее расширение этой схемы за счет включения возмущающих функций высших скейлинговых размерностей позволит получить интегрируемые возмущения для всех членов семейств $\mathrm{AKHC} \mathrm{и} \mathrm{KН,} \mathrm{обладающие} \mathrm{более} \mathrm{богатыми} \mathrm{интегрируемыми} \mathrm{структурами}$ с двойной иерархией и ускоряющимися солитонными решениями.

Мы рассмотрели новое применение системы возмущенных НУШ с двойной иерархией для нелинейных оптических коммуникаций посредством солитонного импульса, распространяющегося через активированную резонансную среду. Такое глубокое понимание интегрируемой структуры для неголономной деформации привело нас к преобразованию иерархии возмущенного НУШ в возмущенное НУШ, уравнение Хироты и НУШ высшего порядка, а иерархии неголономных связей - в связанные системы СИП, от традиционной одиночной до новой кратной системы СИП, которая интерпретируется как многократно активированная резонансная среда. Стало понятно, что в системе НУШ-СИП допускаемая теорией произвольная функция $c(z)$ может регулировать профиль начальной инверсной заселенности, с помощью чего, в свою очередь, можно управлять солитонным импульсом, используя ранее не изученную возможность регулирования ширины и ускорения оптического импульса, распространяющегося через активированную среду.

Сейчас мы работаем над подробным анализом различных аспектов такой возможности, а также схожими применениями возмущенного ДНУШ и надеемся, что результаты будут скоро опубликованы [22]. Кроме того, сейчас мы изучаем использование неголономной связи в некоторых других интегрируемых системах, таких как уравнение $n$-волн и, что более любопытно, в случае высших размерностей $2+1$ или низших размерностей $0+1$. Это составляет интересную программу будущих исследований [23].

Благодарности. Автор выражает искреннюю благодарность за финансовую поддержку и иную помощь организаторам конференции "Нелинейная физика: теория и эксперимент. VI", в частности профессорам Марко Бойти и Флоре Пемпинелли, а также Марии Жерарди.

\section{Список литературы}

[1] A. Kundu (ed.), Classical and Quantum Nonlinear Integrable Systems: Theory and Applications, IOP, Bristol, 2003; M. Boiti, L. Martina, F. Pempinelli (eds.), Nonlinear Evolution Equations and Dynamical Systems, Proc. NEEDS'91, World Sci., Singapore, 1992.

[2] B. A. Kupershmidt, Phys. Lett. A, 372:15 (2008), 2634-2639, arXiv: 0709.3848.

[3] A. Kundu, J. Math. Phys., 50:10 (2009), 102702, 16 pp., arXiv: 0711.0878. 
[4] A. Karasu-Kalkanli, A. Karasu, A. Sakovich, S. Sakovich, R. Turhan, J. Math. Phys., 49:7 (2008), 073516, 10 pp., arXiv: 0708.3247.

[5] J. Lenells, A. S. Fokas, Nonlinearity, 22:1 (2009), 11-27.

[6] A. Kundu, R. Sahadevan, L. Nalinidevi, J. Phys. A, 42:11 (2009), 115213, 13 pp., arXiv: 0811.0924.

[7] A. Kundu, J. Math. Phys., 51:2 (2010), 022901, 17 pp., arXiv: 0910.0383.

[8] A. Kundu, J. Phys. A, 41:49 (2008), 495201, 7 pp., arXiv: 0806.2743.

[9] V.K. Mel'nikov, Phys. Lett. A, 133:9 (1988), 493-496; Inverse Problems, 6:2 (1990), 233-246; 8:1 (1992), 133-147.

[10] J. F. Gomes, G. R. de Melo, L. H. Ymai, A. H. Zimerman, J. Phys. A, 43:39 (2010), 395203, 9 pp., arXiv: 1004.3488.

[11] Z. Qiao, W. Strampp, Physica A, 313:3-4 (2002), 365-380, arXiv: nlin/0201065.

[12] G.P. Agrawal, Fiber-Optic Communication Systems, Wiley, New York, 2002; V. Alwyn, "Fiber-Optic Technologies", Optical Network Design and Implementation, Cisco Press, Indianapolis, 2004; Encyclopedia of Laser Physics and Technology, http://www.rp-photonics.com/encyclopedia.html, Virtual Web-Library, RP Photonics.

[13] G. P. Agrawal, Nonlinear Fiber Optics, Acad. Press, New York, 2006.

[14] L.F. Mollenauer, R. H. Stolen, J. P. Gordon, Phys. Rev. Lett., 45:13 (1980), 1095-1098; A. Hasegawa, F. D. Tappert, Appl. Phys. Lett., 23:3 (1973), 142-144.

[15] S. L. McCall, E. L. Hahn, Phys. Rev. Lett., 18:21 (1967), 908-911; Phys. Rev., 183:2 (1969), 457-485.

[16] G. L. Lamb Jr., Rev. Modern Phys., 43:2 (1971), 99-124.

[17] А. И. Маймистов, Е.А. Манякин, ЖЭТФ, 85:4 (1983), 1177-1181.

[18] M. Nakazawa, E. Yamada, H. Kubota, Phys. Rev. Lett., 66:20 (1991), 2625-2628.

[19] M. Nakazawa, E. Yamada, H. Kubota, Phys. Rev. A, 44:9 (1991), 5973-5987.

[20] S. Kakei, J. Satsuma, J. Phys. Soc. Japan, 63:3 (1994), 885-894.

[21] K. Porsezian, K. Nakkeeran, Phys. Rev. Lett., 74:15 (1995), 2941-2944.

[22] A. Kundu, Hidden possibilities in controlling optical soliton in fiber guided doped resonant medium, arXiv: 1009.2641.

[23] A. Kundu, SIGMA, 6 (2010), 080, 9 pp. 